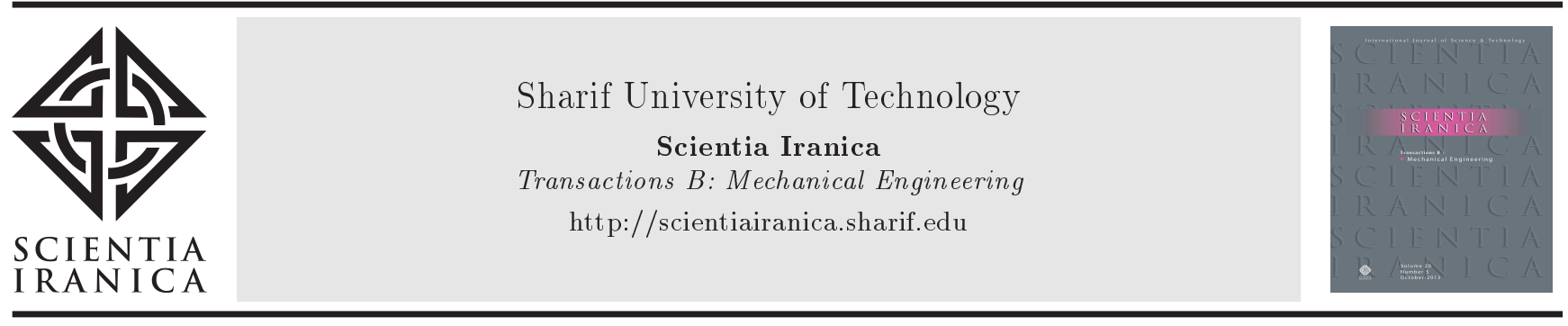

\title{
A new approach to thermo-fluid behavior through porous layer of heat pipes
}

\author{
A. Nouri-Borujerdi* \\ School of Mechanical Engineering, Sharif University of Technology, Tehran, Iran.
}

Received 21 September 2016; received in revised form 3 February 2017; accepted 11 September 2017

\section{KEYWORDS}

Annular condensation

flow;

Heat pipes;

Heat transfer;

Numerical method;

Porous media.

\begin{abstract}
This paper developed a new mathematical model to investigate the heat transfer as well as wick's thickness of a heat pipe. The model was established by conservative equations of continuity, momentum, and energy in the thermal boundary layer. Using a similarity variable, the governing equations were changed to a set of ordinary differential equations and were solved numerically by the forth-order Runge-Kutta method. The flow variables, such as velocity components, wick's thickness, and Nusselt number, were obtained. The results show that the Nusselt number is proportional to the square root of the Darcy-modified Rayleigh number and to the distance from the edge of the condenser surface. Furthermore, the thickness of the wick material depends on the Jakob number and is proportional to the heat transfer between the wall and liquid film.
\end{abstract}

(C) 2018 Sharif University of Technology. All rights reserved.

\section{Introduction}

Heat pipe is a closed thin tube with phase change as a cooling device, as schematically illustrated in Figure 1. There exists an annular wick on the inside surface of the tube. The heat pipe can be constructed from metals, ceramics, composite materials, or glass. It transfers a large amount of heat to remote locations, when the working fluid changes from liquid to vapor at the evaporator side and, then, condenses in the condenser. The fluid is recirculated via capillary pressure in a porous wick lining the evaporator. Heat pipes have many industrial applications in thermal power plants, aerospace applications, and electronic components (not limited to solar energy collectors) so that almost every laptop is equipped at least with one heat pipe, today. Considering the advantages of highly efficient evaporation and condensation heat transfer, heat pipes can achieve excellent temperature unifor-

\footnotetext{
*.E-mail address: anouri@sharif.edu
}

mity with a fast response and high reliability $[1,2]$. The heat pipe performance is primarily governed by wick structure, which provides the capillary pressure for driving the working fluid, flow passage, and heat flow path. Therefore, the thermal performance of heat pipe is strongly dependent on the wick structure, which is important in terms of the porosity, pore size, permeability, and machinability. In addition, its properties, including small-sized pore, high permeability, and low thermal conductivity, as advantages can improve heat transport ability, which is an indicator of the heat pipe performance. The wick material is made of monometallic and mixed metal materials such as cooper, titanium, nickel, stainless steel, etc. to meet the corresponding attribute [3]. There are several typical wick structures such as grooves, sintered mesh or powders, and fibers. The sintered particle wick is generally regarded as the most state-of-art type of heat pipe wick due to its high capillary pressure and moderate-high thermal conductivity. Byon and Kim [4] investigated experimentally and analytically the effects of the particle size distribution and of the packing structure. In this study, they considered two 
distinct types of porous media: mono-dispersed and bi-dispersed. The effect of the particle structure was represented by two geometry parameters: the particle arrangement and the particle-bonded area, depending on the sintering condition. Yang et al. [5] simplified an expression that determines the permeability of isotropic porous media as a function of the tortuosity, porosity, and pore size, thus obtaining an equation that depends only on porosity and pore size. Therefore, this analytical study discusses the effect of these two morphological parameters on the permeability. Tang et al. [6] and Lefévre et al. [7] extensively studied the grooved wicks and mesh wicks. Kempers et al. [8] studied the effect of number of mesh layers on heat pipe performance. They also characterized the evaporation and condensation thermal resistance of mesh-wick heat pipes. The permeability of grooved wicks is high, yet with a limited capability to generate large capillary pressure, while the mesh wicks can generate high capillary pressure, yet suffer from high flow resistance. Hybrid wick structures that can combine the strengths of these two types of wick structures are highly desirable to achieve high performance heat pipes in terms of effective thermal conductivity as well as working load. Powder-groove and mesh-groove wicks are two types of hybrid wicks developed to enhance heat pipe performance [9-11]. The mesh-groove wick with relatively higher permeability is favorable to reduce the flow resistance, particularly with the assistance of gravity. Huang et al. [12] experimentally showed that the partial hybrid wick greatly enhances the heat pipe by $\sim 80 \%$ compared to the heat pipe with a grooved wick. Highly efficient evaporation enabled by the capillary evaporation on the mesh-groove hybrid wick appears to be the primary enhancement mechanism. However, hybrid wicks do not always enhance the heat pipe performance.

Biporous wick is another type of wick which consists of clusters of particles sintered together to form two ranges of pore sizes. One fine pore size present inside the clusters promotes high capillary suctioncapability; further, a coarse pore size present between clusters promotes high permeability for vapor flow, which is essential at high fluxes. For designing a biporous wick, its evaporation characteristics, e.g. evaporative heat transfer coefficient, should be taken into account. So far, Reilly and Catton [13] have relied on kinetic theory of heat transfer by Carey [14], assuming that the liquid-vapor interface exists at saturation temperature. However, the effect of curvature of meniscus has been ignored which is very important at small pore sizes. The effect of meniscus curvature was investigated by Ren et al. [15] on the heat transfer capability of porous wicks at low to moderate heat fluxes. It was shown that their effect is significant, as presented in Kelvin equation.
The purpose of this study is to reveal thermofluid behavior in the wick and optimize the thickness of the wick based on the numerical solution to the governing equations of mass, momentum and energy. Some correlation equations for Nusselt number, wick thickness, and pressure gradient are suggested, where the effects of the Darcy-modified Rayleigh and Jakob numbers are taken into account.

\section{Physical model}

Heat pipe is a closed pipe in which a fluid heat transfer agent circulates naturally between the heating zone or evaporator and the cooling zone or condenser; further, it consists of a sealed container lined with a wicking material (as a porous medium). In this device, the vapor is produced in the evaporator section at the bottom of the pipe and, then, moves upward spontaneously. The vapor is condensed in the condenser section at the top of the pipe and, then, penetrates through the wicking material (see Figure 1).

We assume that this material will be saturated with water, that is, the space between the fibers of wicking material is entirely filled with water, and this water will flow as a result of the gravity and surface tension forces simultaneously imposed on the material. In other words, there will be a free convection flow in the wick. Usually, the saturated flow in the porous media is described by the Darcy model (without viscous term) or by the Brinkman model used in order to account for the high porosity of porous media or to impose no-slip conditions on solid walls. In order to determine the adequate and efficient model, there exists a rule of thumb: the Darcy model is used if the

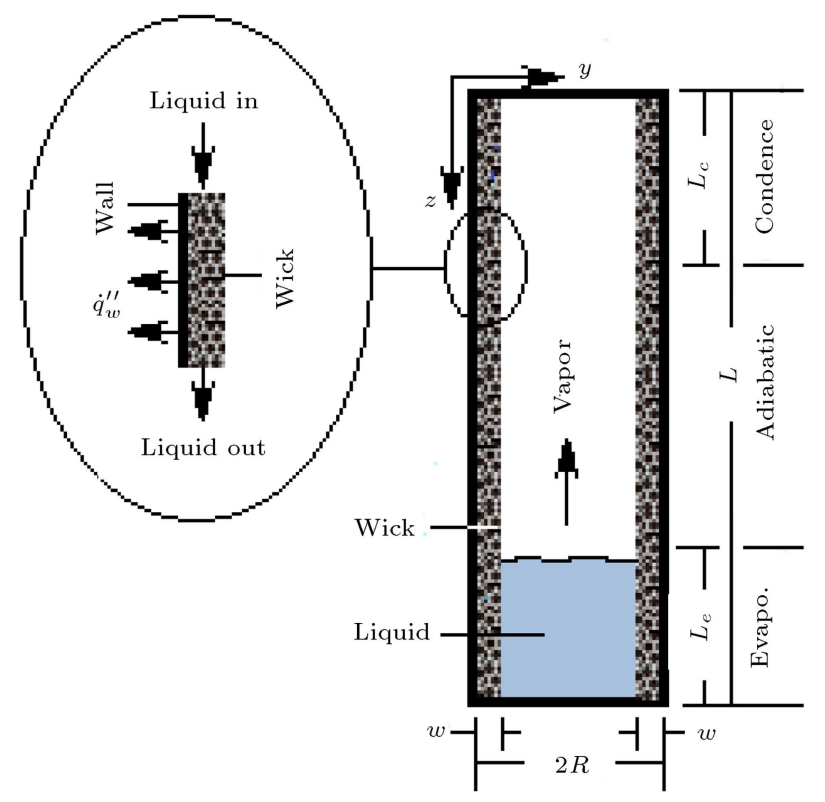

Figure 1. Vapor flow in core and liquid flow in wick lined on the inner surface of a heat pipe. 
Reynolds number of the corresponding free flow is less than $\operatorname{Re}<10$.

Since the wick thickness is very smaller than the radius of the heat pipe, Cartesian coordinates are used instead of cylindrical coordinate system for simplicity. In this case, the Darcy model through the porous layer (wick) in the absence of the inertial force may be obtained as follows:

$$
-\frac{\mu_{f}}{K} u_{f}-\frac{\partial p_{f}}{\partial_{z}}+\rho_{f} g=0
$$

where the viscous term is the usual Darcy term. $K$ denotes the permeability tensor of the wick, $u_{f}$ is the volumetric average of the velocity or Darcy velocity, and $p_{f}$ is the intrinsic fluid pressure. The thickness of the boundary layer is considered herein, in which significant temperature differences exist and velocities parallel to the surface are small. Also:

$$
-\frac{\partial p_{f}}{\partial z}+\rho_{f \mathrm{sat}} g=0
$$

Inserting $\left(\rho-\rho_{f \text { sat }}\right)=\rho \beta /\left(T_{\text {sat }}-T\right)$ and Eq. (2) into Eq. (1) gives:

$$
u_{f}=\frac{g \beta K}{\nu_{f}}\left(T_{\mathrm{sat}}-T\right) .
$$

$T_{\text {sat }}$ is the liquid temperature outside the boundary layer.

By defining a stream function, $\psi$, this satisfies the continuity equation as follows:

$$
\begin{aligned}
& u_{f}=\frac{\partial \psi}{\partial_{y}}, \\
& \nu_{f}=-\frac{\partial \psi}{\partial z} .
\end{aligned}
$$

Because a boundary layer-type flow is assumed, the diffusion in $y$ direction is much greater than that in $x$ direction, and the energy equation can be approximated by:

$$
u_{f} \frac{\partial T}{\partial z}+\nu_{f} \frac{\partial T}{\partial y}=\alpha_{\mathrm{eff}} \frac{\partial^{2} T}{\partial y^{2}}
$$

In order to solve the above equation, we assume that:

$$
\begin{aligned}
& \psi=G(z) F(\eta) \\
& \theta(\eta)=\frac{T_{\mathrm{sat}}-T}{T_{\mathrm{sat}}-T_{w}}=\frac{T_{\mathrm{sat}}-T}{\Delta T}, \\
& \eta=\frac{y}{\delta(z)}
\end{aligned}
$$

where $\delta$ is the boundary layer thickness.
Introducing the unknown variables from Eqs. (4) and (6) into Eqs. (3) and (5), the final results are presented:

$$
\begin{aligned}
& F^{\prime}=\frac{g \beta K \Delta T}{\nu_{f}} \frac{\delta}{G} \theta \\
& \theta^{\prime \prime}+\frac{\delta G^{\prime}}{\alpha_{\mathrm{eff}}} F \theta^{\prime}=0 .
\end{aligned}
$$

Since the two above equations are a function of $\eta$, they are necessary to be constant:

$$
\begin{aligned}
& \frac{g \beta K \Delta T}{\nu_{f}} \frac{\delta}{G}=C_{1}, \\
& \frac{\delta G^{\prime}}{\alpha_{\mathrm{eff}}}=C_{2} .
\end{aligned}
$$

Now, Eqs. (9) and (10) are solved for functions $\delta(z)$ and $G(z)$ with boundary condition $\delta(0)=0$. In this respect, the final results are:

$$
\delta=\left[\frac{2 C_{1} C_{2} \nu_{f} \alpha_{\mathrm{eff}} z}{g \beta K \Delta T}\right]^{0.5}=z \sqrt{\frac{2 C_{1} C_{2}}{\mathrm{Ra}_{z}}},
$$

and:

$$
G=\left[\frac{2 C_{2}}{C_{1}} \frac{g \beta K \Delta T \alpha_{\mathrm{eff}} z}{\nu_{f}}\right]^{0.5}=\alpha_{\mathrm{eff}} \sqrt{\frac{2 C_{2}}{C_{1}} \mathrm{Ra}_{z}}
$$

where $\alpha_{\text {eff }}=k_{\text {eff }} / \rho_{f} C_{p f}$ denotes the effective thermal diffusivity, $\mathrm{Ra}_{z}=g \beta K z \Delta T / \nu_{f} \alpha_{\text {eff }}$ defines the Darcymodified Rayleigh number, and $\Delta T=T_{\text {sat }}-T_{w}$ is a reference difference temperature.

In consequence of Eqs. (4a) and (4b), the velocity components in the direction parallel and normal to the wall are respectively as follows:

$$
\begin{aligned}
& u_{f}=\frac{\alpha_{\text {eff }}}{z} \frac{\operatorname{Ra}_{z}}{C_{1}} F^{\prime}(\eta)=\frac{\alpha_{\text {eff }}}{z} \operatorname{Ra}_{z} \theta(\eta), \\
& \nu_{f}=\frac{\alpha_{\text {eff }}}{2 z} \sqrt{\frac{2 C_{2}}{C_{1}} \operatorname{Ra}_{z}}\left[\eta F^{\prime}(\eta)-F(\eta)\right] .
\end{aligned}
$$

By introducing Eqs. (11) and (12) into Eqs. (7) and (8), the final results will be as follows:

$$
\begin{aligned}
& F^{\prime}-C_{1} \theta=0, \\
& \theta^{\prime \prime}+C_{2} F \theta^{\prime}=0 .
\end{aligned}
$$

Through the combination of the above two equations in favor of function $F(\eta)$, we obtain:

$$
F^{\prime \prime \prime}+C_{2} F F^{\prime \prime}=0
$$


Due to Eqs. (6b), (14), and (15), the requirement boundary conditions are as follows:

$$
\begin{aligned}
& \theta(0)=1, \quad \theta(\infty)=0, \\
& F(0)=0, \quad F^{\prime}(0)=C_{1}, \quad F^{\prime}(\infty)=0 .
\end{aligned}
$$

The local heat transfer on the surface of the condenser will be:

$$
\dot{q}_{w}^{\prime \prime}=-\left.k_{\mathrm{eff}} \frac{\partial T}{\partial y}\right|_{y=0}=k_{\mathrm{eff}} \frac{\theta T}{\delta}(z) \theta^{\prime}(0),
$$

where $k_{\text {eff }}=\phi k_{f}+(1-\phi) k_{s}$ and $\phi=\forall_{f} /\left(\forall_{f}+\forall_{s}\right)$ in which $\forall$ is volume.

The local Nusselt number is calculated as follows:

$$
\mathrm{Nu}_{z}=-\left.\sqrt{\frac{\mathrm{Ra}_{z}}{2 C_{2} C_{1}}} \frac{\partial \theta}{\partial \eta}\right|_{\eta=0}
$$

Further, the mean Nusselt number is then given by:

$$
\overline{\mathrm{Nu}}=\frac{1}{L_{c}} \int_{0}^{L c} \mathrm{Nu}_{z} d z=-\left.2 \sqrt{\frac{\mathrm{Ra}_{L c}}{2 C_{2} C_{1}}} \frac{\partial \theta}{\partial \eta}\right|_{\eta=0},
$$

where $L_{c}$ is the condenser length.

In order to obtain the thickness of the wick, it is necessary for the energy balance between the heat absorbed by the heat sink and that rejected by the core vapor because of condensation to be identical. Therefore, the energy balance between the heat sinking to the condenser surface area $\left(\pi D L_{c}\right)$ and the heat rejected by condensation over the cross-section area of the saturated porous layer $(\pi D w)$ is derived from $z=$ $L_{c}$ in Cartesian coordinates, respectively, as follows:

$$
\left.\int_{0}^{L c} k_{\mathrm{eff}} \pi D \frac{\partial T}{\partial y}\right|_{y=0} d z=\left.\int_{0}^{w} \rho_{f} h_{f g} \pi D u_{f}\right|_{z=L_{c}} d y .
$$

By introducing Eqs. (6b), (6c), (11), and (13) into the above equation, we obtain:

$$
-k_{\mathrm{eff}} \Delta T \theta^{\prime}(0) \int_{0}^{L c} \frac{d z}{\delta}=\rho_{f} h_{f g} \frac{\alpha_{\mathrm{eff}} \mathrm{Ra}_{L c}}{C_{1} L_{c}} \delta\left(L_{c}\right) \int_{0}^{w} F^{\prime} d \eta
$$

After some manipulation, the result is as follows:

$$
F\left[\frac{w}{L_{c}} \sqrt{\mathrm{Ra}}_{L c}\right]=-\left.\frac{\mathrm{Ja}}{C_{2}} \frac{\partial \theta}{\partial \eta}\right|_{\eta=0},
$$

where $\mathrm{Ja}=C_{p} \Delta T / h_{f g}=C_{p}\left(T_{\text {sat }}-T_{w}\right) / h_{f g}$ is the Jakob number. For a specific value of Jakob number and the value $\partial \theta /\left.\partial \eta\right|_{\eta=0}=-0.443$ from Figure 6 , the value of the wick thickness can be obtained by function of $F\left(w \sqrt{\mathrm{Ra}_{L c}} / L_{c}\right)$ from Figure 8 .

\section{Solution method}

Eqs. (15) and (16) as a pair of simultaneous ordinary differential equations are first combined, and a third-order ordinary differential equation is obtained with respect to $F$ (Eq. (17)). This equation needs three boundary conditions. The first two boundary conditions are $F^{\prime}(0)=C_{1} \theta(0)=C_{1}$, and $F^{\prime}(\infty)=C_{1} \theta(\infty)=0$ and the third one is obtained from the liquid normal velocity $\nu_{f}=$ $\alpha_{\text {eff }} \sqrt{2 C_{2} \operatorname{Ra}_{z} / C_{1}}\left[\eta F^{\prime}(\eta)-F(\eta)\right] / 2 z$ at the wall which is zero. Eq. (17) is a two-point boundary value problem and is necessary to be changed into three first-order initial-value problems with initial conditions $F(0)=0$, $F^{\prime}(0)=C_{1}$ and a guessed value for $F^{\prime \prime}(0)=A$. Direct integration using Runge-Kutta is only possible until a guessed initial value for $A$ is provided and keeps getting corrected and updated along the integration process until condition $F^{\prime}(\infty)=0$ is satisfied; otherwise, a new value for $A$ is guessed and the previous procedure is iterated again. After solving the functions of $F, F^{\prime}$, and $F^{\prime \prime}$ the other functions such as $\theta, \theta^{\prime}$ and stream function, $\psi$, are obtained. Once the functions of $\psi$ and $\theta$ are determined, the flow variables, such as velocity components, local Nusselt number, and wick thickness, are obtained from Eqs. (6a), (13), (14), (20) and (24), respectively.

\section{Results and discussion}

A closed two-phase water-vapor heat pipe made of a thin copper tube with length of adiabatic, $L_{a}=0.4 \mathrm{~m}$ and those of condenser and evaporator $L_{c}=L_{e}=0.3$ $\mathrm{m}$ of has been considered. The wick material of the heat pipe is made of wire crimps with porosity of $\phi=0.7$ and unknown thickness of $w$ determined by Eq. (24). It should be noted that $C_{1}$ and $C_{2}$ are constant in all above equations so that they can be either chosen arbitrarily or fused into functions of $G(z)$ and $\delta(z)$, respectively, in Eqs. (9) and (10). In order to determine the correct value of $C_{2}$ Eq. (17) has been solved numerically by the fourth-order Runge-Kutta method under the boundary conditions of Eq. (18b) for different values of $C_{2}$. Figure 2 shows that the proper behavior of function $F(\eta)$ is obtained when $C_{1}=1$ and $C_{2}=0.05$.

Figure 3 depicts function $F(\eta)$ and its first and second derivatives versus similarity parameter $\eta$. The values of $F(\eta)$ and $F^{\prime \prime}(\eta)$ reach 1.61 and -0.443 when eta approach 6 and zero, respectively. In addition, function $F(\eta)$ can be approximated by a polynomial function of degree four as follows:

$$
\begin{aligned}
F(\eta)= & -1.6 \times 10^{-3} \eta^{4}+3.25 \times 10^{-2} \eta^{3} \\
& -0.264 \eta^{2}+1.022 \eta-0.0024
\end{aligned}
$$




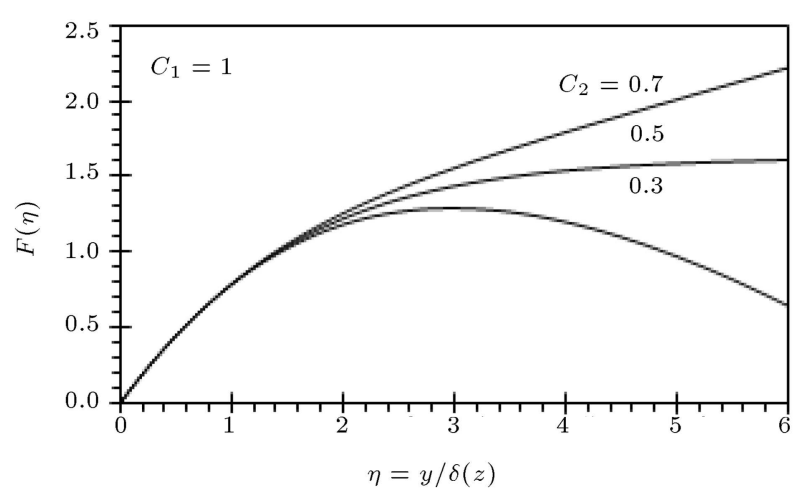

Figure 2. Variation of function $F(\eta)$ versus $\eta$ for different values of $C_{2}$ in Eq. (17).

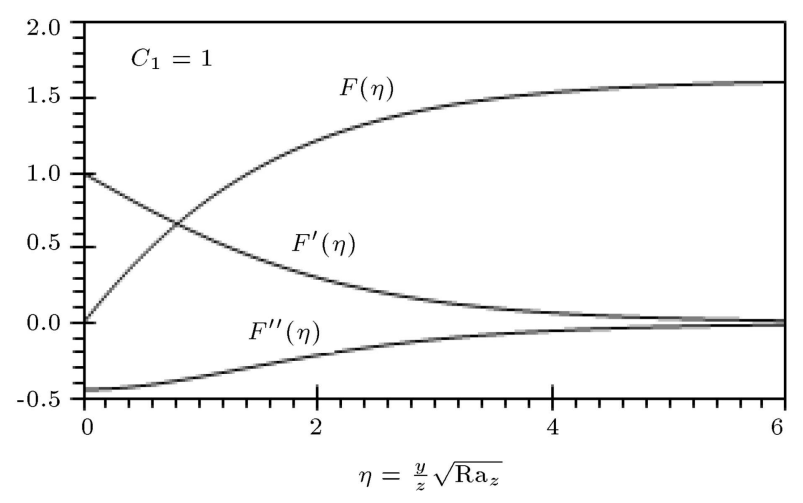

Figure 3. Variations of $F(\eta), F^{\prime}(\eta)$, and $F^{\prime \prime}(\eta)$ versus similarity parameter $\eta$.

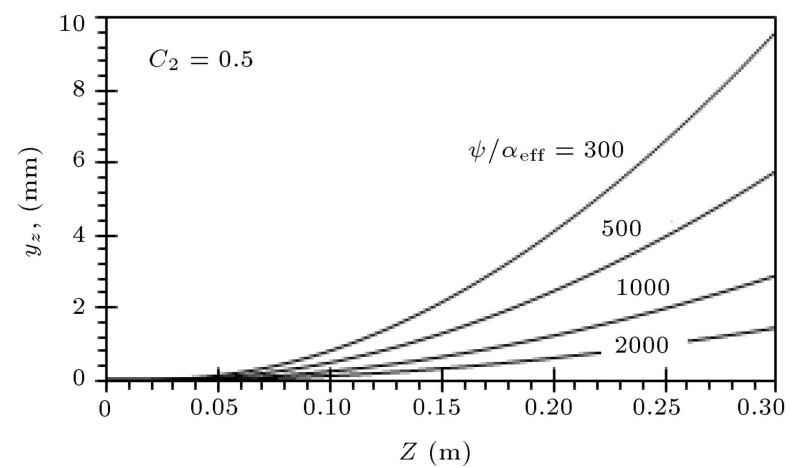

Figure 4. Different stream functions along the condenser length.

Figure 4 illustrates the stream function of the liquid film along the condenser length for four different values of $\psi / \alpha_{\text {eff }}=\sqrt{2 C_{2} \mathrm{Ra}_{z} / C_{1}} F(\eta)=2 C_{2} \eta F(\eta) z / y=$ $300,500,1000$, and 2000 . In this figure, the stream function changes monotonically and decreases in $y$ direction at any level of the condenser length. The corresponding values of the similarity parameter are in the range of 0 to 6 . Interestingly, by moving upward, the slope of the curves increases and the flow tends towards a two-dimensional flow.

Figure 5 exhibits the variation of the velocity components of $u_{f}$ and $\nu_{f}$ in the direction parallel and

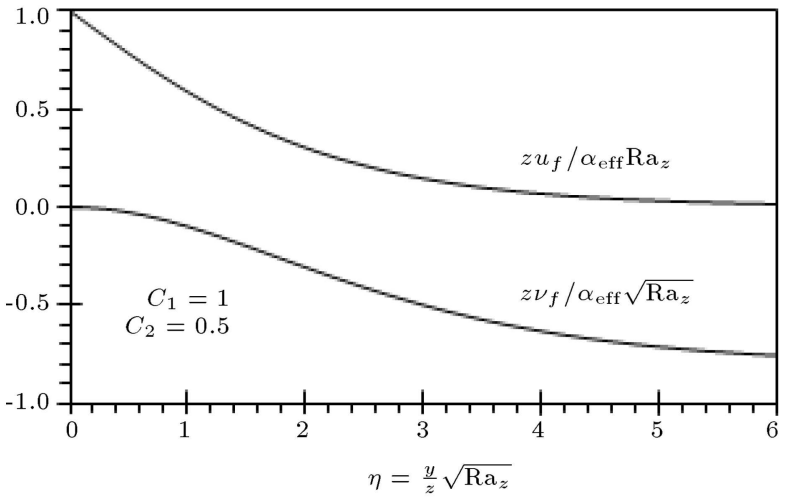

Figure 5. Velocity components of $u_{f}$ and $\nu_{f}$ versus similarity parameter $\eta$.

normal to the wall of heat pipe, respectively. The scale of $u_{f}$ is more significant in comparison to that of $\nu_{f}$, and the ratio between them is proportional to the square root of the Rayleigh number, i.e. $u_{f} / \nu_{f} \propto$ $\mathrm{Ra}_{z}^{0.5}$. Furthermore, normal velocity, $\nu_{f}$, starts with zero at the wall due to lack of penetration and reaches a maximum value of $-0.757 \alpha_{\text {eff }} \sqrt{\mathrm{Ra}_{z}} / z$ at $\eta=6$.

Figure 6 illustrates the non-dimensional temperature distribution of the liquid film, $\theta=\left(T_{\text {sat }}-\right.$ $T) /\left(T_{\text {sat }}-T_{w}\right)$, as a function of similarity parameter, $\eta$. The results indicate that the temperature of the liquid film approaches the saturation temperature at the edge of the thermal boundary layer. In other words, at this location, the rate of phase change from vapor to liquid is nearly zero and reaches a maximum value at the wall. In addition, function $\theta(\eta)$ can be approximated by a polynomial function of degree four as follows:

$$
\begin{aligned}
\theta(\eta)= & -10^{-4} \eta^{4}-4 \times 10^{-3} \eta^{3}+8.6 \\
& \times 10^{-2} \eta^{2}-0.506 \eta+1.01
\end{aligned}
$$

Figure 7 exhibits the local Nusselt number against the condenser length. The correlation of the Nusselt number based on Eq. (20) and $\partial \theta /\left.\partial \eta\right|_{\eta=0}=-0.443$ from Figure 6 is $\mathrm{Nu}_{z} / \sqrt{\mathrm{Ra}_{L_{c}}}=0.443 \sqrt{z / L_{c}}$, and

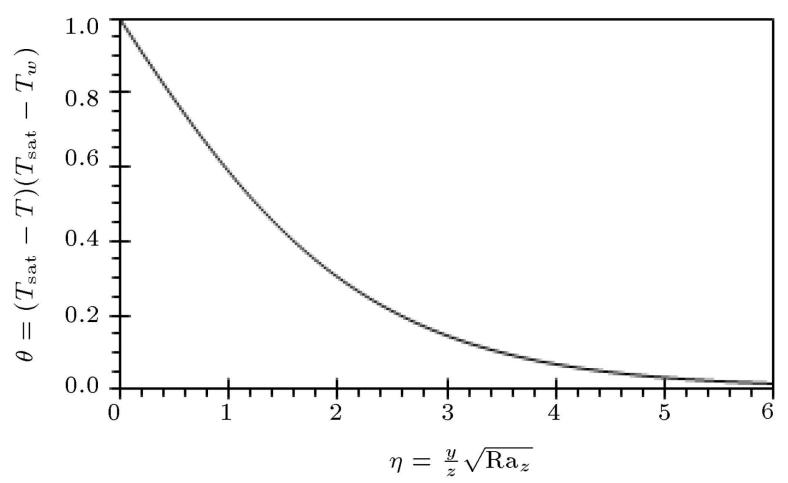

Figure 6. Temperature distribution of liquid film in the porous layer. 


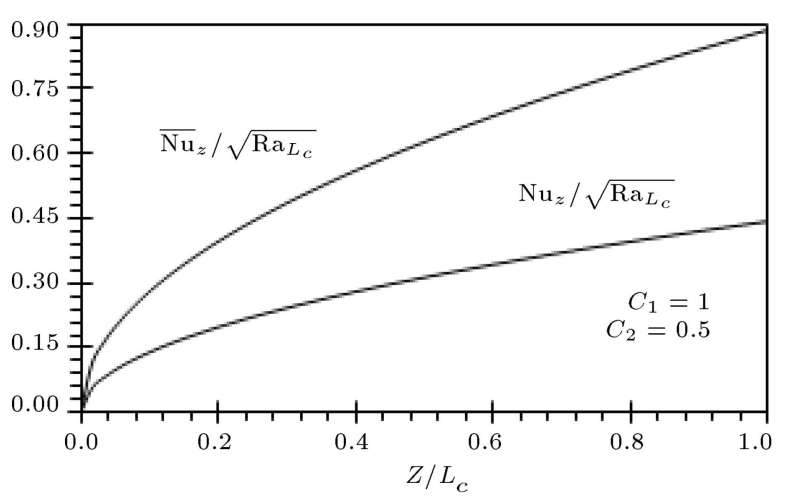

Figure 7. Local and averaged Nusselt numbers along the condenser length.

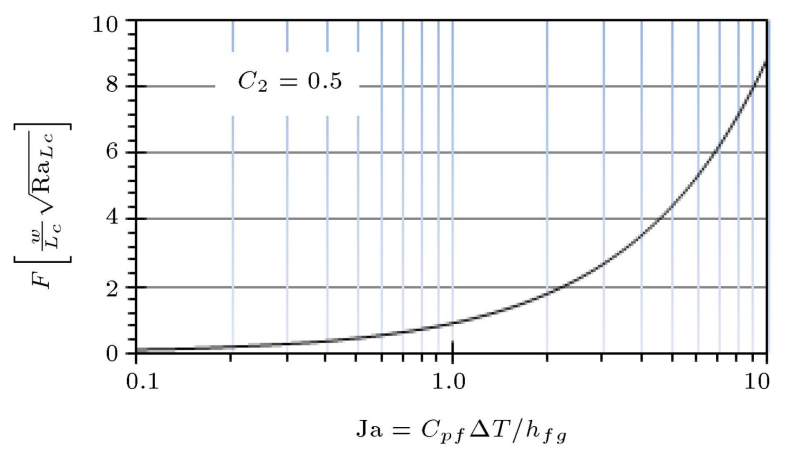

Figure 8. Function of wick thickness versus the Jakob number.

the corresponding average Nusselt number is given by $\overline{\mathrm{Nu}_{z}} / \sqrt{\mathrm{Ra}_{L_{c}}}=0.886 \sqrt{z / L_{c}}$.

Figure 8 shows the variation of wick thickness versus Jakob number. It is found that the required wick thickness of a heat pipe for refrigerant and organic working fluids is greater than that of the water, because the Jakob number of water vaporization is smaller than those of the other fluids. On the other hand, the Jakob number is inversely proportional to the latent heat of vaporization.

\section{Conclusions}

This paper developed a mathematical model to simulate the liquid film in the wick of a heat pipe. The model was established by the governing equations in the thermal boundary layer. The results reveal that the local Nusselt number was correlated through $\mathrm{Nu}_{z}=0.443 \sqrt{\mathrm{Ra}_{z}}$, and the thickness of the wick material depends on the Jakob number as well as the modified Rayleigh number. On the other hand, the wick thickness of a heat pipe for refrigerant and organic working fluids is larger than that of the water. The reason is that the Jakob number is inversely proportional to the latent heat of vaporization so that the refrigerant latent heat of vaporization is smaller than that of the water.

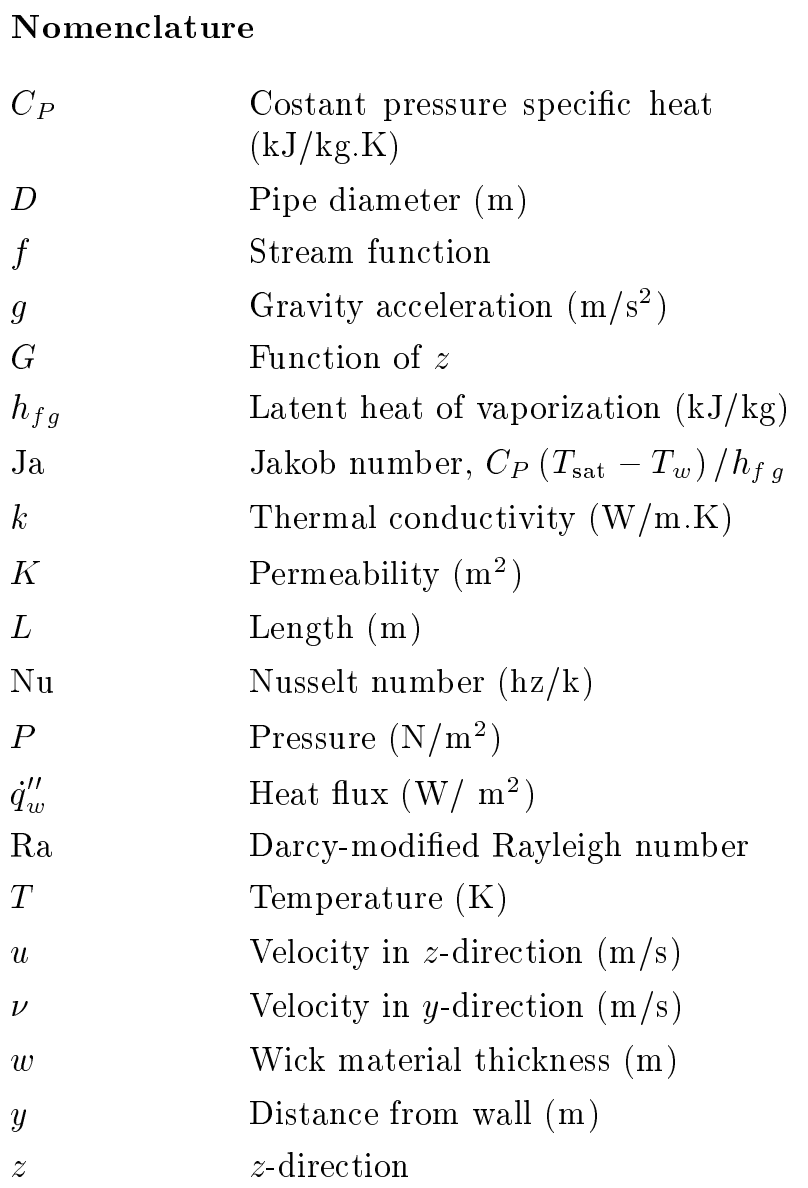

\section{Greek letters}

$\alpha$

Molecular thermal diffusivity $\left(\mathrm{m}^{2} / \mathrm{s}\right)$

$\beta$

$\delta$

$\eta$

$\Delta$

$\phi$

$\mu$

$\nu$

$\theta$

$\psi$

$\rho$

$\forall$

\section{Subscripts}

$c$

eff

$f$

$s$

Expansion coefficient $(1 / \mathrm{K})$

Film thickness (m)

Similarity parameter, $y / \delta$

Difference

Porosity

Viscosity $(\mathrm{kg} / \mathrm{m} . \mathrm{s})$

Kinematic viscosity $\left(\mathrm{m}^{2} / \mathrm{s}\right)$

Non-dimensional, $\left(T_{\text {sat }}-T\right) /\left(T_{\text {sat }}-T_{w}\right)$

Stream function, $g(z) f(\eta)$

Density $\left(\mathrm{kg} / \mathrm{m}^{3}\right)$

Volume $\left(\mathrm{m}^{3}\right)$

Condenser

Effective

Liquid

Solid

Saturation

Wall

\section{Superscripts}

Average 


\section{References}

1. Chi, S.W., Heat Pipe Theory and Practice: a Sourcebook (Series in Thermal and Fluids Engineering), McGraw-Hill Inc., USA (1976).

2. Peterson, G.P., An Introduction to Heat Pipes: Modeling, Testing, and Applications, John Wiley \& Sons, Inc., New York (1994).

3. Chung, W.B., Hwang, S.H., Park, C.M., Kim, Y.S. and Kim, S.S., Development of Loop Heat Pipes with c Using Cold Isostatic Press Method, Joint 18th IHPC and 12th IHPS, Jeju, Korea, June 12-16 (2016).

4. Byon, C. and Kim, S.J. "Permeability of mono- and bi-dispersed porous media", EPJ Web of Conferences, 45, 01018 (2013).

5. Yang, X., Lu, T.J. and Kim, T. "An analytical model for permeability of isotropic porous media", Physics Letters A., 378(30-31), pp. 2308-2311 (2014).

6. Tang, Y., Hu, Z., Qing, J., Xie, Z., Fu, T., and Chen, W. "Experimental investigation on isothermal performance of the micro-grooved heat pipe", Experimental Thermal and Fluid Science, 47, pp. 143-149, (2013).

7. Lefèvre, F., Conrardy, J.B., Raynaud, M., and Bonjour, J. "Experimental investigations of flat plate heat pipes with screen meshes or grooves covered with screen meshes as capillary structure", Applied Thermal Engineering, 37, pp. 95-102, (2012).

8. Kempers, R., Ewing, D., and Ching, C.Y. "Effect of number of mesh layers and fluid loading on the performance of screen mesh wicked heat pipes", Applied Thermal Engineering, 26(5-6), pp. 589-595 (2006).

9. Jiang, L., Ling, J., Jiang, L., Tang, Y., Li, Y., Zhou, W., and Gao, J. "Thermal performance of a novel porous crack composite wick heat pipe", Energy Conversion and Management, 81, pp. 10-18 (2014).

10. Hsieh, J.C., Huang, H.J., and Shen, S.C. "Experimental study of micro rectangular groove structure covered with multi mesh layers on performance of flat plate heat pipe for LED lighting module", Microelectronics Reliability, 52(6), pp. 1071-1079 (2012).

11. Dai, X., Famouri, M., Abdulagatov, A.E., Yang, R., Lee, Y.C., George, S.M., and Li, C. "Capillary evaporation on micro membrane-enhanced microchannel wicks with atomic layer deposited silica", Applied Physics Letters, $103(15)$, p. 151602 (2013).

12. Huang, G., Abdulshaheed, A., Chang, W., and Li, C. "An evaluation of hybrid wick design on high performance copper-ethanol heat pipes", Joint 18th IHPC and 12th IHPS, Jeju, Korea, June 12-16 (2016).

13. Reilly, S., and Catton, I. "Utilization of pore-size distributions to predict thermophysical properties and performance of biporous wick evaporators", Journal of Heat Transfer, ASME, 136(6), 061501, (2014).

14. Carey, V.P. Liquid-Vapor Phase-Change Phenomena, Hemisphere, New York (1992).

15. Ren, C., Wu, Q.S., and Hu, M.B. "Heat transfer with flow and evaporation in loop heat pipe's wick at low or moderate heat fluxes", Int. J. of Heat Mass Trans., Elsevier, 50(11-12), pp. 2296-2308 (2007).

\section{Biography}

Ali Nouri-Borujerdi is a Professor of Mechanical Engineering at Sharif University of Technology, Tehran, Iran, where he teaches undergraduate and graduate level courses in the Thermal/Fluids Science. His teaching focuses on heat transfer, computational fluid dynamics, and two-phase flows. His current research programs include computational, analytical, and experimental study of heat and mass transfer, porous media, transport phenomena, fuel cells, and twophase flows with boiling and condensation in industries applications. Professor Nouri has published more than 150 articles in international journals and conferences. 